\title{
DETERMINATION OF SPECIFIC RESISTANCE OF HIGHLY COMPRESSED CAKE BY USE OF EXPRESSION DATA
}

\author{
TOSHIRO MURASE, MASASHI IWATA, ICHIO KATO, \\ WON-DEOK LEE AND MOMPEI SHIRATO \\ Department of Chemical Engineering, Nagoya University, Nagoya 464
}

\begin{abstract}
Key Words: Solid Liquid Separation, Specific Resistance, Expression, Highly Compressed Cake, Semisolid Material
\end{abstract}

Specific resistance $\alpha$, which is one of the fundamental properties of particulate beds, is usually determined by the compression permeability test. ${ }^{1,4)}$ However, this method is rather tedious, especially in determining an $\alpha$-value of highly compressed cake, where a liquid should be permeated into the cake for a long time. A previous paper $^{2)}$ presented a new technique for obtaining permeability data in a moderate pressure range based on a filtration experiment. In this paper, we shall present a convenient method for obtaining a local $\alpha$-value of highly compressed cake, using both numerical solutions of the consolidation equation and empirical expression data of homogeneous semisolid materials.

\section{Theory}

If the creep effect of the material is negligible, the consolidation equation can be represented by the form $^{3)}$

$$
\begin{aligned}
& \frac{\partial p_{s}}{\partial \theta_{c}}=C_{e}\left\{\frac{\partial^{2} p_{s}}{\partial \omega^{2}}-\frac{1}{\alpha}\left(\frac{d \alpha}{d p_{s}}\right)\left(\frac{\partial p_{s}}{\partial \omega}\right)^{2}\right\} \\
& C_{e}=\frac{1}{\mu \rho_{s} \alpha\left(-d e / d p_{s}\right)}
\end{aligned}
$$

where $p_{s}$ is the local solid compressive pressure; $\theta_{c}$, the consolidation time; $C_{e}$, the local modified consolidation coefficient and a function of $p_{s}$; $\omega$, the volume of solid per unit sectional area between an arbitrary position in cake and the filter medium; $\alpha$, the local specific resistance of cake; $\mu$, the viscosity of liquid; $\rho_{s}$, the true density of solid, and $e$, the local void ratio of cake. Eq. (1) can be rewritten by using the following variables:

$$
\begin{gathered}
\phi=p_{s} / p \\
z=i \omega / \omega_{0} \\
T_{c}^{*}=i^{2} C_{e}^{*} \theta_{c} / \omega_{0}^{2}
\end{gathered}
$$

Received July 1, 1987. Correspondence concerning this article should be addressed to T. Murase.

$$
C=C_{e} \frac{p}{\alpha} \frac{d \alpha}{d p_{s}}
$$

in a dimensionless form as

$$
\frac{\partial \phi}{\partial T_{c}^{*}}=\frac{C_{e}}{C_{e}^{*}} \frac{\partial^{2} \phi}{\partial z^{2}}-\frac{C}{C_{e}^{*}}\left(\frac{\partial \phi}{\partial z}\right)^{2}
$$

Here $p$ is the expression pressure; $i$, the number of drainage surfaces; $\omega_{0}$, the total solid volume in cake per unit sectional area; $T_{c}^{*}$, the dimensionless consolidation time; and $C_{e}^{*}$, the local $C_{e}$-value at the drainage surface $\left(p_{s}=p\right)$.

If compression permeability data of $\alpha$ and $e$ can be represented in the following form:

$$
\begin{gathered}
\alpha=\alpha_{0} p_{s}^{n} \\
e=E_{0}-C_{c} \ln p_{s}
\end{gathered}
$$

then Eq. (7) can be rewritten in the form

$$
\frac{\partial \phi}{\partial T_{c}^{*}}=\phi^{1-n} \frac{\partial^{2} \phi}{\partial z^{2}}-n \phi^{-n}\left(\frac{\partial \phi}{\partial z}\right)^{2}
$$

Numerical solutions of Eq. (10) are summarized in terms of the average consolidation ratio, $U_{c}$, which is a measure of the extent of consolidation.

$$
U_{c}=\frac{\int_{0}^{1}\left(e_{1}-e\right) d z}{\int_{0}^{1}\left(e_{1}-e_{\infty}\right) d z}=\frac{\int_{0}^{1} \ln \left(p_{s} / p_{s 1}\right) d z}{\int_{0}^{1} \ln \left(p / p_{s 1}\right) d z}
$$

Here $e_{1}$ denotes the initial void ratio of homogeneous semisolid material; $e_{\infty}$, the equilibrium void ratio of compressed cake under the expression pressure $p$; and $p_{s 1}$, the pre-consolidation pressure for obtaining a homogeneous semisolid material of void ratio $e_{1}$. Since the solution $\phi$ of Eq. (10) is a function of $T_{c}^{*}, z$, $n$ and $p / p_{s 1}$, the $U_{c}-T_{c}^{*}$ relation of semisolid material depends only on the values of $n$ and $p / p_{s 1}$.

Based on Eqs. (10) and (11), the dimensionless consolidation time $T_{c 50}^{*}$ required for attaining $50 \%$ of $U_{c}$ at each $n$ and $p / p_{s 1}$ is shown in Fig. 1. In the 


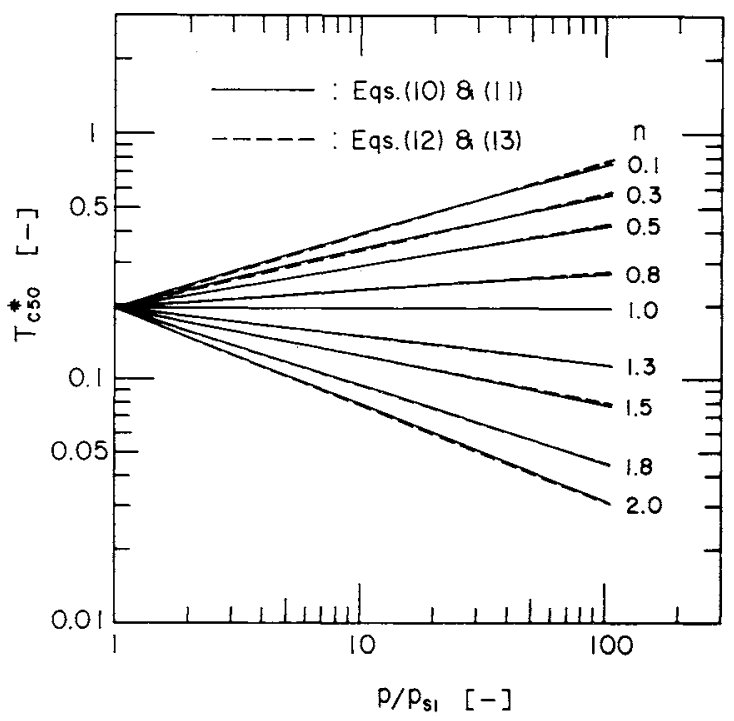

Fig. 1. Relationship between $T_{c 50}^{*}, p / p_{s 1}$ and $n$

numerical computations of Eq. (10), the interval of dimensionless distance $\Delta z$ was set at 0.1 and that of dimensionless time $\Delta T_{c}^{*}$ was set at 0.0001 . Since the relation between $\log T_{c 50}^{*}$ and $\log \left(p / p_{s 1}\right)$ at each $n$ is almost linear, as shown in the figure, they can be represented by the following formula.

$$
\begin{gathered}
T_{c 50}^{*}=0.196\left(p / p_{s 1}\right)^{f(n)} \\
f(n)=-0.0379 n^{2}-0.288 n+0.324 \\
\left(0.1 \leqq n \leqq 2.0, \quad 2 \leqq p / p_{s 1} \leqq 100\right)
\end{gathered}
$$

On the other hand, combining Eqs. (2), (5), (8) and (9), $T_{c 50}^{*}$ is expressed by

$$
T_{c 50}^{*}=\frac{i^{2} \theta_{c 50} p_{s 1}^{1-n}}{\mu \omega_{0}^{2} \rho_{s} \alpha_{0} C_{c}}\left(p / p_{s 1}\right)^{1-n}
$$

where $0_{c 50}$ is the real consolidation time for attaining $50 \%$ of $U_{c}$. Therefore, combining Eqs. (12) and (14) yields

$$
\frac{i^{2} \theta_{c 50} p_{s 1}^{1-n}}{\mu \omega_{0}^{2} \rho_{s} \alpha_{0} C_{c}}=0.196\left(p / p_{s 1}\right)^{f(n)+n-1}
$$

That is, for semisolid materials at the same preconsolidation pressure $p_{s 1}$,

$$
\frac{i^{2} \theta_{c 50}}{\mu \omega_{0}^{2}} \propto\left(p / p_{s 1}\right)^{f(n)+n-1}
$$

because $\rho_{s}, C_{c}, \alpha_{0}, n$ and $p_{s 1}$ are constant for these materials. Accordingly, obtaining $\theta_{c 50}$ from constantpressure expression data, and plotting $\left(i^{2} \theta_{c 50} / \mu \omega_{0}^{2}\right)$ and $\left(p / p_{s 1}\right)$ on logarithmic graph paper, we can find the value of $n$ from the slope of the plots.

$$
\begin{aligned}
\text { slope } & =f(n)+n-1 \\
& =-0.0379 n^{2}+0.712 n-0.676
\end{aligned}
$$

The local specific resistance, $\alpha$, corresponding to the

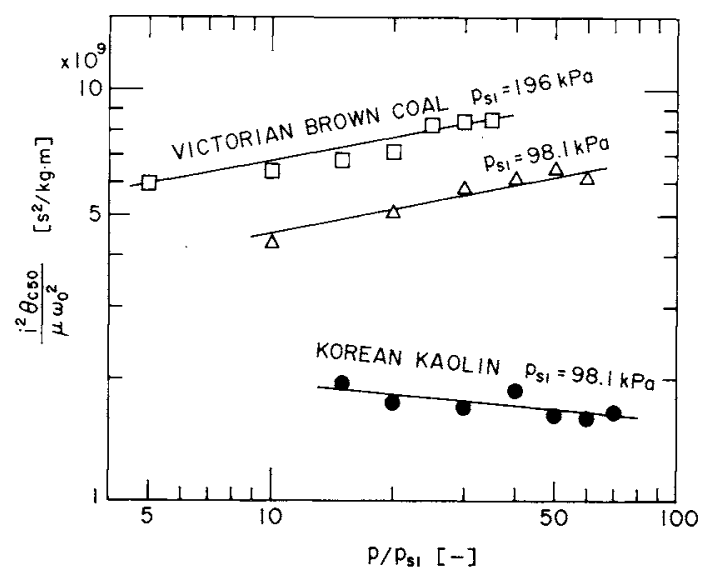

Fig. 2. Empirical determination of $n$

expression pressure $p$, is obtained from Eqs. (2), (5), (9) and (12), thus:

$$
\alpha=\frac{i^{2} \theta_{\mathrm{c} 50} p}{\mu \omega_{0}^{2} \rho_{s} C_{c}\left\{0.196\left(p / p_{s 1}\right)^{f(n)}\right\}}
$$

\section{Discussion}

The experimental materials used in this study were Korean kaolin $\left(\rho_{s}=2600 \mathrm{~kg} / \mathrm{m}^{3}\right)$ and Victorian brown coal $\left(\rho_{s}=1480 \mathrm{~kg} / \mathrm{m}^{3}\right)$. These materials were slurried and placed in a compression permeability cell. ${ }^{1,4}$ Compression permeability data $\alpha$ and $e$ were measured ${ }^{1,4)}$ under solid compressive pressure $p_{s}$ of $981 \mathrm{kPa}-6.86 \mathrm{MPa} .\left(C_{c}=0.161\right.$ for Korean kaolin and 0.458 for brown coal.) These slurried materials were also pre-consolidated under pressure $p_{s 1}$ of 98.1$196 \mathrm{kPa}$, resulting in homogeneous semisolid materials. Then these semisolid materials were expressed under constant-pressure $p$ of $981 \mathrm{kPa}-6.86 \mathrm{MPa}$, thereby obtaining $\theta_{c 50}$.

Figure 2 shows the empirical relation between $\left(i^{2} 0_{c 50} / \mu \omega_{0}^{2}\right)$ and $\left(p / p_{s 1}\right)$. It can be seen from the figure that the slopes of plots of Victorian brown coal are independent of the pre-consolidation pressure $p_{s 1}$. From the slopes of plots in the figure, we can find that $n=0.88$ for Korean kaolin and 1.30 for Victorian brown coal. Figure 3 shows the $\alpha-p_{s}$ relation calculated from Eq. (18). The agreement between calculated and experimental $\alpha$-values (obtained by the permeability test) is satisfactory. (The maximum error of the calculated value compared to the experimental one is $18 \%$ for Korean kaolin and $35 \%$ for Victorian brown coal.)

A future research topic would be to obtain the $\alpha$ value of material which fails to obey Eqs. (8) and (9).

\section{Acknowledgment}

We would like to express our thanks to Dr. P. J. Banks of CSIRO for furnishing Victorian brown coal used in the experiments. 


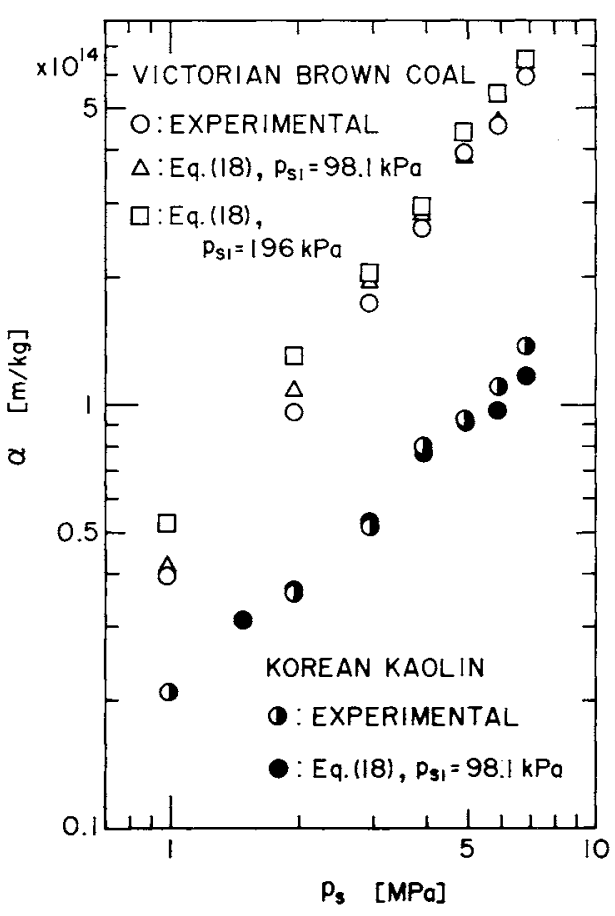

Fig. 3. Comparison of theoretical and experimental permeability data

\section{Nomenclature}

$C_{c} \quad=$ empirical constant for compression data

$C_{e} \quad=$ local modified consolidation coefficient

$C_{e}^{*}=C_{e}$-value at the drainage surface $\left(p_{s}=p\right)$

$E_{0} \quad=$ empirical constant for compression data

$e \quad=$ local void ratio

$e_{\mathrm{i}} \quad=$ initial void ratio $e_{\infty} \quad=$ equilibrium void ratio under expression pressure $p$

$=$ number of drainage surfaces

$=$ compressibility coefficient

$=$ expression pressure

$p_{s} \quad=$ local solid compressive pressure equilibrium void ratio $e_{1}$ is attained

$=$ dimensionless consolidation time

$=$ dimensionless consolidation time for attaining $50 \%$ of $U_{c}$

$U_{c} \quad=$ average consolidation ratio

$z \quad=$ dimensionless distance

$=$ local specific resistance

$=$ empirical constant for permeability data

$=$ consolidation time

$\theta_{c 50}=$ consolidation time for attaining $50 \%$ of $U_{c}$

$=$ viscosity of liquid

$=$ true density of solid

$=$ volume of solid per unit sectional area between an arbitrary position in cake and a filter medium

$=$ total solid volume in cake per unit sectional area

$[\mathrm{m}]$

\section{Literature Cited}

1) Grace, H. P.: Chem. Eng. Progr., 49, 303, 367 (1953).

2) Murase, T., E. Iritani, J. H. Cho, S. Nakanomori and M. Shirato: J. Chem. Eng. Japan, 20, 246 (1987)

3) Shirato, M., T. Murase, M. Negawa and H. Moridera: $J$. Chem. Eng. Japan, 4, 263 (1971).

$\left[\mathrm{m}^{2} / \mathrm{s}\right]$

$[-]$

$[-]$

[-]
4) Shirato, M.: "Kagaku Kogaku Benran," 4th ed., p. 1140, Maruzen (1978). 